\title{
COEFFICIENTS OF PROLONGATIONS FOR SYMMETRIES OF ODES
}

\author{
RICARDO ALFARO and JIM SCHAEFERLE
}

Received 4 September 2003

\begin{abstract}
Sophus Lie developed a systematic way to solve ODEs. He found that transformations which form a continuous group and leave a differential equation invariant can be used to simplify the equation. Lie's method uses the infinitesimal generator of these point transformations. These are symmetries of the equation mapping solutions into solutions. Lie's methods did not find widespread use in part because the calculations for the infinitesimals were quite lengthy, needing to calculate the prolongations of the infinitesimal generator. Nowadays, prolongations are obtained using Maple or Mathematica, and Lie's theory has come back to the attention of researchers. In general, the computation of the coefficients of the $(n)$ prolongation is done using recursion formulas. Others have given methods that do not require recursion but use Fréchet derivatives. In this paper, we present a combinatorial approach to explicitly write the coefficients of the prolongations. Besides being novel, this approach was found to be useful by the authors for didactical and combinatorial purposes, as we show in the examples.
\end{abstract}

2000 Mathematics Subject Classification: 34C14, 34A25, $22 \mathrm{E} 99$.

1. Introduction. For an ODE (written in the form $\Delta=0$ ), in the independent variable $x$ and the dependent variable $y$, we define a one-parameter group of point transformations $\tilde{x}=\tilde{x}(x, y, \epsilon)$ and $\tilde{y}=\tilde{y}(x, y, \epsilon)$, where for $\epsilon=0$ we have the identity transformation. The infinitesimal generator for this group of transformations is defined as the vector field $X=\xi(x, y) \partial x+\eta(x, y) \partial y$, where $\xi(x, y)=\partial \tilde{x} /\left.\partial \epsilon\right|_{\epsilon=0}$ and $\eta(x, y)=\partial \tilde{y} /\left.\partial \epsilon\right|_{\epsilon=0}$. Lie's theory is based on calculating these infinitesimal generators. To do this, we need to transform the derivatives $y^{(k)}$ in the ODE to obtain $\tilde{y}^{(k)}$. This is obtained, equivalently, by prolonging the infinitesimal generator to

$$
X=\xi \partial x+\eta \partial y+\eta^{(1)} \partial y^{\prime}+\cdots+\eta^{(m)} \partial y^{(m)},
$$

where the coordinates are given by $\eta^{(k)}:=\partial \tilde{y}^{(k)} /\left.\partial \epsilon\right|_{\epsilon=0}$.

Normally, the coefficients of the prolongation are obtained recursively by the formula

$$
\eta^{(n)}=\frac{d}{d x} \eta^{(n-1)}-y^{(n)} \frac{d \xi}{d x}
$$

where $d / d x$ represents the total derivative (see [3, page 12]). The prolongation of the vector field can then be expressed as a linear combination of differential monomials of the form $\left(y^{\prime}\right)^{a_{1}}\left(y^{\prime \prime}\right)^{a_{2}} \ldots\left(y^{(m)}\right)^{a_{m}}$. 
The coefficients of the derivative monomials that occur will produce the "determining equations" (see [1]), obtained by solving

$$
\left(\xi \partial x+\eta \partial y+\eta^{(1)} \partial y^{\prime}+\cdots+\eta^{(m)} \partial y^{(m)}\right)(\Delta) \equiv 0(\bmod \Delta) .
$$

These equations form a system of partial differential equations, and its solutions form a Lie group of point transformations, which determine the symmetries of the original ODE. Each symmetry can be used to simplify the equation to one of lower order. See [2, 3] for further details of these methods. The calculation by hand of these coefficients can be tedious especially as the order of the ODE increases, and are generally obtained by recursion algorithms on computer algebra software. We present here a direct formula for the coefficients of these monomials, using a combinatorial approach.

2. The formulas. First, using a direct induction argument, we convert the recursive expression of $\eta^{(n)}$ above to an expression in terms of total derivatives.

LEMMA 2.1. The nth coefficient in the prolongation of the infinitesimal operator is given by

$$
\eta^{(n)}=\frac{d^{n} \eta}{d x^{n}}-\sum_{i=1}^{n}\left(\begin{array}{c}
n \\
i
\end{array}\right) \frac{d^{(n+1-i)} y}{d x^{(n+1-i)}} \cdot \frac{d^{i} \xi}{d x^{i}}
$$

Proof. For $n=1$, we obtain the original definition in (1.2). Assume the formula holds for $n-1$. By (1.2) and use of the product rule, we have

$$
\begin{aligned}
\eta^{(n)}= & \frac{d \eta^{(n-1)}}{d x}-\frac{d^{n} y}{d x^{n}} \frac{d \xi}{d x} \\
= & \frac{d}{d x}\left(\frac{d^{(n-1)} \eta}{d x^{(n-1)}}-\sum_{i=1}^{n-1}\left(\begin{array}{c}
n-1 \\
i
\end{array}\right) \frac{d^{(n-i)} y}{d x^{(n-i)}} \cdot \frac{d^{i} \xi}{d x^{i}}\right)-\frac{d^{n} y}{d x^{n}} \frac{d \xi}{d x} \\
\eta^{(n)}= & \frac{d^{n} \eta}{d x^{n}}-\sum_{i=1}^{n-1}\left(\begin{array}{c}
n-1 \\
i
\end{array}\right) \frac{d^{(n+1-i)} y}{d x^{(n+1-i)}} \cdot \frac{d^{i} \xi}{d x^{i}} \\
& -\sum_{i=1}^{n-1}\left(\begin{array}{c}
n-1 \\
i
\end{array}\right) \frac{d^{(n-i)} y}{d x^{(n-i)}} \cdot \frac{d^{(i+1)} \xi}{d x^{(i+1)}}-\frac{d^{n} y}{d x^{n}} \frac{d \xi}{d x} .
\end{aligned}
$$

After a change of indices, we obtain

$$
\begin{aligned}
\eta^{(n)}= & \frac{d^{n} \eta}{d x^{n}}-\sum_{i=1}^{n-1}\left(\begin{array}{c}
n-1 \\
i
\end{array}\right) \frac{d^{(n+1-i)} y}{d x^{(n+1-i)}} \cdot \frac{d^{i} \xi}{d x^{i}} \\
& -\sum_{i=0}^{n-2}\left(\begin{array}{c}
n-1 \\
i
\end{array}\right) \frac{d^{(n-i)} y}{d x^{(n-i)}} \cdot \frac{d^{(i+1)} \xi}{d x^{(i+1)}}-\frac{d y}{d x} \frac{d^{n} \xi}{d x^{n}}
\end{aligned}
$$




$$
\begin{aligned}
\eta^{(n)} & =\frac{d^{n} \eta}{d x^{n}}-\sum_{i=1}^{n-1}\left(\left(\begin{array}{c}
n-1 \\
i
\end{array}\right)+\left(\begin{array}{c}
n-1 \\
i-1
\end{array}\right)\right) \frac{d^{(n+1-i)} y}{d x^{(n+1-i)}} \cdot \frac{d^{i} \xi}{d x^{i}}-\frac{d y}{d x} \frac{d^{n} \xi}{d x^{n}} \\
& =\frac{d^{n} \eta}{d x^{n}}-\sum_{i=1}^{n-1}\left(\begin{array}{c}
n \\
i
\end{array}\right) \frac{d^{(n+1-i)} y}{d x^{(n+1-i)}} \cdot \frac{d^{i} \xi}{d x^{i}}-\frac{d y}{d x} \frac{d^{n} \xi}{d x^{n}} \\
& =\frac{d^{n} \eta}{d x^{n}}-\sum_{i=1}^{n}\left(\begin{array}{c}
n \\
i
\end{array}\right) \frac{d^{(n+1-i)} y}{d x^{(n+1-i)}} \cdot \frac{d^{i} \xi}{d x^{i}} .
\end{aligned}
$$

DEFINITION 2.2. The $i$ th power order of a monomial of derivatives of the form $\left(y^{\prime}\right)^{a_{1}}\left(y^{\prime \prime}\right)^{a_{2}} \cdots\left(y^{(n)}\right)^{a_{n}}$, where $a_{j} \geq 0, j=1, \ldots, n$, is defined as the integer

$$
s_{i}=s_{i}\left(\left(y^{\prime}\right)^{a_{1}}\left(y^{\prime \prime}\right)^{a_{2}} \cdots\left(y^{(n)}\right)^{a_{n}}\right):=: \sum_{j=i}^{n} a_{j} .
$$

The $J$-order of the monomial is defined as the integer

$$
q=q\left(\left(y^{\prime}\right)^{a_{1}}\left(y^{\prime \prime}\right)^{a_{2}} \cdots\left(y^{(n)}\right)^{a_{n}}\right):=: \sum_{i=1}^{n} s_{i}
$$

Note that $s_{1}$ is the usual degree of the monomial, and the $J$-order is equal to $q=$ $\sum_{i=1}^{n} s_{i}=\sum_{i=1}^{n} i a_{i}$. We present an expression for the $n$th total derivative in terms of the derivative monomials as follows.

Proposition 2.3. The expansion of the $n$th total derivative with respect to $x$ for $a$ differential operator in two variables $x, y$, where $y$ is the dependent variable, is given by

$$
\frac{d^{n}}{d x^{n}}=\sum_{\substack{\left(a_{1}, a_{2}, \ldots, a_{n}\right) \\ a_{i} \geq 0, q \leq n}} \frac{n !}{(n-q) ! \prod_{i=1}^{n}\left(a_{i} ! i^{s_{i}}\right)} \cdot\left(y^{\prime}\right)^{a_{1}} \cdots\left(y^{(n)}\right)^{a_{n}} \cdot \partial x^{(n-q)} \partial y^{s_{1}},
$$

where $q$ is the corresponding $J$-order.

Proof. For $n=1$, we have $s_{1}=a_{1}$, and the possible tuples in the summation are (0) and (1):

$$
\begin{aligned}
\sum_{\left(a_{1}\right)=(0),(1)} & \frac{1 !}{\left(1-s_{1}\right) ! a_{1} ! 1^{s_{1}}}\left(y^{\prime}\right)^{a_{1}} \partial x^{\left(1-s_{1}\right)} \partial y^{s_{1}} \\
& =\frac{1 !}{1 ! 0 ! 1^{0}} \partial x+\frac{1 !}{0 ! 1 ! 1^{1}} y^{\prime} \partial y=\partial x+y^{\prime} \partial y=\frac{d}{d x} .
\end{aligned}
$$


Now, using the total derivative definition, we have

$$
\begin{aligned}
\frac{d^{n}}{d x^{n}} & =\frac{d}{d x}\left(\frac{d^{n-1}}{d x^{n-1}}\right) \\
& =\left(\partial x+y^{\prime} \partial y+y^{\prime \prime} \partial y^{\prime}+\cdots+y^{(n)} \partial y^{(n-1)}\right) \cdot\left(\frac{d^{n-1}}{d x^{n-1}}\right)
\end{aligned}
$$

An arbitrary term in the expansion of $d^{n} / d x^{n}$ with respect to the derivatives in the $y$ variable will be of the form

$$
\alpha \cdot\left(y^{\prime}\right)^{b_{1}}\left(y^{\prime \prime}\right)^{b_{2}} \cdots\left(y^{(n)}\right)^{b_{n}}
$$

and let $s_{i}$ be the corresponding power orders of the term. The idea is to look at the possible ways this term could have appeared in the expansion of (2.8). We pair up the terms in the total derivative part with the corresponding terms in the $(n-1)$ th total derivative part, which can add to our particular term in $d^{n} / d x^{n}$. Depending on our arbitrary term, some terms cannot be paired, so we have to add existence conditions to the terms.

By the induction hypothesis and the nature of the terms in the first factor in (2.8), the restrictions on the choices of exponents are $b_{i} \geq 0$ for all $i$ and $q=\sum_{i=1}^{n} s_{i} \leq n$, where $s_{i}=\sum_{j=i}^{n} b_{j}$. Notice that this condition gives only two choices for $b_{n}$, namely, 0 or 1 , and in the case $b_{n}=1$ we have $b_{i}=0$ for all $i=1, \ldots, n-1$. We will consider the two cases separately.

CASE $1\left(b_{n}=1, b_{i}=0\right.$ for all $\left.i=1, \ldots, n-1\right)$. The only way we can obtain this term is by applying $y^{(n)} \partial_{y^{(n-1)}}$ to the last term in the expansion of the $(n-1)$ th derivative. In this case, $s_{i}=1$ for all $i$ and $q=n$. Thus

$$
\begin{aligned}
& \alpha \cdot\left(y^{\prime}\right)^{0} \cdots\left(y^{(n-1)}\right)^{0} \cdots\left(y^{(n)}\right)^{1} \\
& \quad=y^{(n)} \partial_{y^{(n-1)}}\left(\frac{(n-1) !}{1} \frac{1}{(n-1) !} \cdot\left(y^{(n-1)}\right) \cdot \partial y\right)=y^{(n)} \partial y .
\end{aligned}
$$

On the other hand, for this case,

$$
\frac{n !}{(n-q) ! \prod\left(b_{i} ! i^{s_{i}}\right)} \partial x^{n-\sum s_{i}} \partial y=\partial y=\alpha
$$

CASE $2\left(b_{n}=0\right)$. We look at the possible "sources" for the term $\alpha \cdot\left(y^{\prime}\right)^{b_{1}}\left(y^{\prime \prime}\right)^{b_{2}}$ $\cdots\left(y^{(n-1)}\right)^{b_{n-1}}$ to occur in the expansion of (2.8).

For clarity, we have sometimes dropped the limits in the summation and product, and it is understood what the limits are from the context of the tuples in the formula. Thus

$$
\alpha \cdot\left(y^{\prime}\right)^{b_{1}} \cdots\left(y^{(n-1)}\right)^{b_{n-1}}=\sum_{j=0}^{n-1} \alpha_{j},
$$


where

$$
\begin{gathered}
\alpha_{0}=\partial_{x}\left(\frac{(n-1) !}{\left((n-1)-\sum r_{i}\right) !} \frac{1}{\prod\left(b_{i} ! i^{r_{i}}\right)} \cdot\left(y^{\prime}\right)^{b_{1}} \cdots\left(y^{(n-1)}\right)^{b_{n-1}} \cdot \partial x^{(n-1)-\sum r_{i}} \partial y^{r_{1}}\right), \\
\alpha_{1}=\left\{\begin{array}{cc}
y^{\prime} \partial_{y}\left(\frac{(n-1) !}{(n-1)-\left(\sum r_{i}-1\right) !} \frac{b_{1}}{\prod\left(b_{i} ! i^{r_{i}}\right)}\right. & \text { if } b_{1}=0, \\
\left.\cdot\left(y^{\prime}\right)^{b_{1}-1}\left(y^{\prime \prime}\right)^{b_{2}} \cdots\left(y^{(n-1)}\right)^{b_{n-1}} \cdot \partial x^{\left((n-1)-\left(\sum r_{i}-1\right)\right)} \partial y^{r_{1}-1}\right) & \text { if } b_{1}>0,
\end{array}\right. \\
\alpha_{j}=\left\{\begin{array}{cc}
y^{(j)} \partial_{y^{(j-1)}\left(\frac{(n-1) !}{(n-1)-\left(\sum r_{i}-1\right) !} \frac{1}{\prod\left(b_{i} ! i^{r_{i}}\right)} \frac{j b_{j}}{b_{j-1}+1}\right.}^{\cdot\left(y^{\prime}\right)^{b_{1}} \cdots\left(y^{(j-1)}\right)^{b_{j-1}+1}\left(y^{(j)}\right)^{b_{j}-1} \cdots\left(y^{(n-1)}\right)^{b_{n-1}}} \\
\left.\cdot \partial x^{\left((n-1)-\left(\sum r_{i}-1\right)\right)} \partial y^{r_{1}}\right) & \text { if } b_{j}>0, \\
0 & \text { if } b_{j}=0 .
\end{array}\right.
\end{gathered}
$$

The term $r_{i}$ denotes the $i$ th power order of the term in the expansion form of the $(n-1)$ th total derivative, and there are $n-1$ of them, hence the summation goes from 1 to $n-1$. Then $s_{i}=r_{i}$ for $i=1, \ldots, n-1$ and $s_{n}=0$. Thus $\sum_{i=1}^{n-1} r_{i}=\sum_{i=1}^{n} s_{i}$, and the $J$-degrees coincide. We make these changes in the above expressions, and in $\alpha_{0}$ we multiply and divide by $n-q$. The conditions in each formula can then be dropped, giving

$$
\begin{aligned}
& \alpha_{0}=\partial_{x}\left(\frac{(n-1) !(n-q)}{(n-q) !} \frac{1}{\prod\left(b_{i} ! i^{s_{i}}\right)} \cdot\left(y^{\prime}\right)^{b_{1}} \cdots\left(y^{(n-1)}\right)^{b_{n-1}} \cdot \partial x^{((n-1)-q)} \partial y^{s_{1}}\right), \\
& \alpha_{1}=y^{\prime} \partial_{y}\left(\frac{(n-1) !}{(n-q) !} \frac{b_{1}}{\prod\left(b_{i} ! i^{s_{i}}\right)} \cdot\left(y^{\prime}\right)^{b_{1}-1}\left(y^{\prime \prime}\right)^{b_{2}} \cdots\left(y^{(n-1)}\right)^{b_{n-1}} \cdot \partial x^{(n-q)} \partial y^{s_{1}}\right), \\
& \alpha_{j}=y^{(j)} \partial_{y^{(j-1)}}\left(\frac{(n-1) !}{(n-q) !} \frac{1}{\prod\left(b_{i} ! i^{s_{i}}\right)} \frac{j b_{j}}{b_{j-1}+1}\right. \\
& \left.\cdot\left(y^{\prime}\right)^{b_{1}} \cdots\left(y^{(j-1)}\right)^{b_{j-1}+1}\left(y^{(j)}\right)^{b_{j}-1} \cdots\left(y^{(n-1)}\right)^{b_{n-1}} \cdot \partial x^{(n-q)} \partial y^{s_{1}}\right) .
\end{aligned}
$$

Applying the differential operators in each case, we obtain

$$
\begin{aligned}
\alpha_{0}= & \frac{(n-1) !(n-q)}{\left(n-\sum s_{i}\right) !} \frac{1}{\prod\left(b_{i} ! i^{s_{i}}\right)} \cdot\left(y^{\prime}\right)^{b_{1}} \cdots\left(y^{(n-1)}\right)^{b_{n-1}} \cdot \partial x^{(n-q)} \partial y^{s_{1}}, \\
\alpha_{1}= & \frac{(n-1) !}{(n-q) !} \frac{b_{1}}{\prod\left(b_{i} ! i^{s_{i}}\right)} \cdot\left(y^{\prime}\right)^{b_{1}}\left(y^{\prime \prime}\right)^{b_{2}} \cdots\left(y^{(n-1)}\right)^{b_{n-1}} \cdot \partial x^{(n-q)} \partial y^{s_{1}} \\
\alpha_{j}= & \frac{(n-1) !}{(n-q) !} \frac{1}{\prod\left(b_{i} ! i_{i}\right)} \frac{j b_{j}}{b_{j-1}+1} \frac{b_{j-1}+1}{1} \\
& \cdot\left(y^{\prime}\right)^{b_{1}} \cdots\left(y^{(j-1)}\right)^{b_{j-1}}\left(y^{(j)}\right)^{b_{j}} \cdots\left(y^{(n-1)}\right)^{b_{n-1}} \cdot \partial x^{(n-q)} \partial y^{s_{1}}
\end{aligned}
$$


Collecting all terms $\alpha_{j}$ for $j=0, \ldots, n-1$, we have

$$
\begin{aligned}
& \alpha \cdot\left(y^{\prime}\right)^{b_{1}} \cdots\left(y^{(n-1)}\right)^{b_{n-1}} \\
& \quad=\frac{(n-1) !\left(n-q+\sum j b_{j}\right)}{(n-q) ! \prod\left(b_{i} ! i^{s_{i}}\right)} \cdot\left(y^{\prime}\right)^{b_{1}} \cdots\left(y^{(n-1)}\right)^{b_{n-1}} \cdot \partial x^{(n-q)} \partial y^{s_{1}} .
\end{aligned}
$$

In the formula above we can extend the product of derivatives to include $\left(y^{(n)}\right)^{b_{n}}$ since $b_{n}=0$. We also have $q=\sum_{i=1}^{n} s_{i}=\sum_{i=1}^{n-1} r_{i} \leq n-1$. The only case where this sum is equal to $n$ has been dealt with in Case 1 , so we can rewrite the condition as $q \leq n$. Hence, the formula above can be written as

$$
\begin{aligned}
& \alpha \cdot\left(y^{\prime}\right)^{b_{1}}\left(y^{\prime \prime}\right)^{b_{2}} \cdots\left(y^{(n)}\right)^{b_{n}} \\
& \quad=\frac{n !}{(n-q) ! \prod\left(b_{i} ! i^{s_{i}}\right)} \cdot\left(y^{\prime}\right)^{b_{1}} \cdots\left(y^{(n)}\right)^{b_{n}} \cdot \partial x^{(n-q)} \partial y^{s_{1}},
\end{aligned}
$$

where $b_{i} \geq 0, q \leq n$.

We now use Proposition 2.3 in the formula from Lemma 2.1 to get a descriptive formula for the prolongation of the vector field in terms of the derivative monomials.

THEOREM 2.4. For the vector field $X=\xi \partial x+\eta \partial y$, the $(m)$ th prolongation

$$
X=\xi \partial x+\eta \partial y+\eta^{(1)} \partial y^{\prime}+\cdots+\eta^{(m)} \partial y^{(m)}
$$

satisfies, for $n=1, \ldots, m$,

$$
\begin{aligned}
\eta^{(n)}= & \sum_{\substack{\left(b_{1}, b_{2}, \ldots, b_{n}\right) \\
b_{i} \geq 0, q \leq n}} \frac{n !}{(n-q) ! \prod_{i=1}^{n}\left(b_{i} ! i^{r_{i}}\right)} \cdot\left(y^{\prime}\right)^{b_{1}} \cdots\left(y^{(n)}\right)^{b_{n}} \cdot \eta_{x^{n-q} y^{r_{1}}} \\
& -\sum_{\substack{\left(b_{1}, b_{2}, \ldots, b_{n}\right) \\
b_{i} \geq 0, q \leq n+1}} \frac{n ! q}{(n+1-q) ! \prod\left(b_{i} ! i^{r_{i}}\right)} \cdot\left(y^{\prime}\right)^{b_{1}} \cdots\left(y^{(n)}\right)^{b_{n}} \cdot \xi_{x^{n+1-q_{y}} y^{r_{1}-1}},
\end{aligned}
$$

where $r_{i}$ is the ith power order and $q$ is the J-order of each monomial.

Proof. We apply Proposition 2.3 to the $\eta$ and $\xi$ parts of the formula given in Lemma 2.1.

The $\eta$ part gives

$$
\frac{d^{n} \eta}{d x^{n}}=\sum_{\substack{\left(b_{1}, b_{2}, \ldots, b_{n}\right) \\ b_{i} \geq 0, \sum_{i=1}^{n} r_{i} \leq n}} \frac{n !}{\left(n-\sum_{i=1}^{n} r_{i}\right) ! \prod_{i=1}^{n}\left(a_{i} ! i^{r_{i}}\right)} \cdot\left(y^{\prime}\right)^{b_{1}} \cdots\left(y^{(n)}\right)^{b_{n}} \cdot \eta_{x^{n-\sum r_{i}} y^{r_{1}}},
$$

where $r_{i}=\sum_{k=i}^{n} b_{k}$. 
The $\xi$ part gives

$$
\begin{aligned}
& \sum_{j=1}^{n}\left(\begin{array}{l}
n \\
j
\end{array}\right) \frac{d^{(n+1-j)} y}{d x^{(n+1-j)}} \cdot \frac{d^{j} \xi}{d x^{j}} \\
& =\sum_{j=1}^{n} \sum_{\left(a_{1}, a_{2}, \ldots, a_{j}\right)}\left(\begin{array}{l}
n \\
j
\end{array}\right) \frac{j !}{\left(j-\sum_{i=1}^{j} s_{i}\right) ! \prod_{i=1}^{j}\left(a_{i} ! i^{s_{i}}\right)} \\
& a_{i} \geq 0, \sum_{i=1}^{j} s_{i} \leq j \\
& \cdot\left(y^{\prime}\right)^{a_{1}} \cdots\left(y^{(j)}\right)^{a_{j}}\left(y^{(n+1-j)}\right) \cdot \xi_{x^{j-\sum s_{i}} y^{s_{1}}} .
\end{aligned}
$$

We use a change of index as follows:

$$
\begin{array}{ll}
b_{i}=a_{i} & \text { for } 1 \leq i \leq j, i \neq n+1-j, \\
b_{i}=0 & \forall j<i \leq n, i \neq n+1-j, \\
b_{i}=1 & \text { for } i=n+1-j .
\end{array}
$$

If $n+1>2 j$, we have $\left(b_{1}, \ldots, b_{n}\right)=\left(a_{1}, \ldots, a_{j}, 0, \ldots, 1,0, \ldots, 0\right)$, where 1 occurs in position $n+1-j$; if $n+1 \leq 2 j$, we have $\left(b_{1}, \ldots, b_{n}\right)=\left(a_{1}, \ldots, a_{n+1-j}+1, \ldots, a_{j}, 0, \ldots, 0\right)$. Let $r_{i}=\sum_{k=i}^{n} b_{i}$, and express

$$
\prod_{i=1}^{j}\left(a_{i} ! i^{s_{i}}\right)=\frac{\prod_{i=1}^{n}\left(b_{i} ! i^{r_{i}}\right)}{b_{n+1-j}(n+1-j) !} .
$$

We now justify that the change of index can be made simultaneously for all the terms in the summation, and thus the condition $\sum_{i=1}^{j} s_{i} \leq j$ is equivalent to the condition $\sum_{i=1}^{n} r_{i} \leq n+1$ for each term. Notice that the term is 0 if $b_{n+1-j}=0$, and we can assume $b_{n+1-j}>0$.

The change of index gives us in both cases

$$
\sum_{i=1}^{n} r_{i}=\sum_{i=1}^{n} i b_{i}=\sum_{i=1}^{j} i a_{j}+(n+1-j)=(n+1-j)+\sum_{i=1}^{j} s_{i} .
$$

We check that no other tuples (except the ones given by the change of index) appear. Consider the tuples for which $n+1-j>j$. Since $\sum_{i=1}^{n} r_{i} \leq n+1$, we necessarily have $b_{n+1-j}=1$. Furthermore, if some $b_{i} \neq 0$ for some $i>j$, then $\sum_{i=1}^{n} r_{i} \geq(n+1-j) \cdot 1+i>$ $n+1$, a contradiction. Hence $b_{i}=0$ for all $i>j, i \neq n+1-j$. Now, consider the tuples for which $n+1-j \leq j$, then $\sum_{i=1}^{n} r_{i}-(n+1-j) \leq(n+1)-(n+1-j)=j$; and since $\sum_{i=1}^{n} r_{i}-(n+1-j)=\sum_{i=1}^{j} s_{i}+\sum_{i=j+1}^{n} i b_{i}$, we obtain $b_{i}=0$ for all $i>j$. We can then simultaneously change the condition for the tuples in the summation

$$
\sum_{i=1}^{n} r_{i} \leq n+1 \Longleftrightarrow \sum_{i=1}^{j} s_{i} \leq j
$$


Performing these changes and using (2.24), (2.23), (2.21) can be expressed as

$$
\begin{aligned}
& \sum_{j=1}^{n} \sum_{\substack{\left(b_{1}, b_{2}, \ldots, b_{n}\right) \\
b_{i} \geq 0, \sum_{i=1}^{n}, r_{i} \leq n+1}}\left(\begin{array}{c}
n \\
j
\end{array}\right) \frac{j !(n+1-j) ! b_{n+1-j}}{\left(j-\left(\sum_{i=1}^{n} r_{i}-(n+1-j)\right)\right) ! \prod_{i=1}^{n}\left(b_{i} ! i^{r_{i}}\right)} \\
& \cdot\left(y^{\prime}\right)^{b_{1}} \cdots\left(y^{(n)}\right)^{b_{n}} \cdot \xi_{x^{n+1-\sum r_{i} y^{r_{1}-1}}} \\
& =\sum_{\substack{\left(b_{1}, b_{2}, \ldots, b_{n}\right) \\
b_{i} \geq 0, \sum_{i=1}^{n}, r_{i} \leq n+1}} \frac{n !}{\left(n+1-\sum_{i=1}^{n} r_{i}\right) ! \prod_{i=1}^{n}\left(b_{i} ! i^{r_{i}}\right)} \cdot\left(\sum_{j=1}^{n}(n+1-j) b_{n+1-j}\right) \\
& \cdot\left(y^{\prime}\right)^{b_{1}} \cdots\left(y^{(n)}\right)^{b_{n}} \cdot \xi_{x^{n+1-\sum r_{i}} y^{r_{1}-1}} \\
& =\sum_{\substack{\left(b_{1}, b_{2}, \ldots, b_{n}\right) \\
b_{i} \geq 0, \sum_{i=1}^{n} r_{i} \leq n+1}} \frac{n ! \sum r_{i}}{\left(n+1-\sum r_{i}\right) ! \prod\left(b_{i} ! i^{r_{i}}\right)} \cdot\left(y^{\prime}\right)^{b_{1}} \cdots\left(y^{(n)}\right)^{b_{n}} \cdot \xi_{x^{n+1-\sum r_{i}} y^{r_{1}-1}} .
\end{aligned}
$$

Now we combine both parts (2.20) and (2.26) to obtain

$$
\begin{aligned}
\eta^{(n)}= & \sum_{\substack{\left(b_{1}, b_{2}, \ldots, b_{n}\right) \\
b_{i} \geq 0, \sum_{i=1}^{n} r_{i} \leq n}} \frac{n !}{\left(n-\sum_{i=1}^{n} r_{i}\right) ! \prod_{i=1}^{n}\left(b_{i} ! i^{r_{i}}\right)} \cdot\left(y^{\prime}\right)^{b_{1}} \cdots\left(y^{(n)}\right)^{b_{n}} \cdot \eta_{x^{n-\sum r_{i}} y^{r_{1}}} \\
& -\sum_{\substack{\left(b_{1}, b_{2}, \ldots, b_{n}\right) \\
b_{i} \geq 0, \sum_{i=1}^{n} r_{i} \leq n+1}} \frac{n ! \sum r_{i}}{\left(n+1-\sum r_{i}\right) ! \prod\left(b_{i} ! i^{\left.r_{i}\right)}\right.} \cdot\left(y^{\prime}\right)^{b_{1}} \cdots\left(y^{(n)}\right)^{b_{n}} \cdot \xi_{x^{n+1-\sum r_{i}} y^{r_{1}-1}},
\end{aligned}
$$

where $r_{i}=\sum_{k=i}^{n} b_{k}$. If we use $q=q\left(b_{1}, \ldots, b_{n}\right)=\sum r_{i}$, we obtain formula (2.19).

COROLLARY 2.5. Equation (2.19) can be written alternatively as

$$
\eta^{(n)}=\sum_{\substack{\left(b_{1}, b_{2}, \ldots, b_{n}\right) \\ b_{i} \geq 0 \\ q=\sum_{i=1}^{n} r_{i} \leq n+1}} \frac{n !}{(n+1-q) !} \prod_{i=1}^{n} \frac{\left(y^{(i)}\right)^{b_{i}}}{b_{i} ! i^{r_{i}}} \cdot\left[(n+1-q) \eta_{x^{n-q} y^{r_{1}}}-(q) \xi_{x^{n+1-q} y^{r_{1}-1}}\right] .
$$

EXAMPLE 2.6. Consider the second-order ODE $y^{\prime \prime}=\left(y^{\prime}\right)^{3}$. The second prolongation of the infinitesimal vector field is

$$
X=\xi \partial x+\eta \partial y+\eta^{(1)} \partial y^{\prime}+\eta^{(2)} \partial y^{\prime \prime}
$$

We apply this prolongation to the equation $y^{\prime \prime}-\left(y^{\prime}\right)^{3}=0$, and get

$$
\eta^{(2)}-3\left(y^{\prime}\right)^{2} \eta^{(1)}=0 .
$$


The determining equations for the symmetries are obtained by making the coefficients of the terms $\left(y^{\prime}\right)^{n}$ equal to 0 , since the functions $\xi, \eta$ depend only on $x, y$. We apply formula (2.19).

For $\eta^{(2)}$, we consider tuples $\left(a_{1}, a_{2}\right)$ such that $q=a_{1}+2 a_{2} \leq 3$; see Table 2.1.

TABLE 2.1

\begin{tabular}{cccc}
\hline Tuple & Term & $\eta$ coefficient & $\xi$ coefficient \\
\hline$a_{1}=0, a_{2}=1$ & $y^{\prime \prime}=\left(y^{\prime}\right)^{3}$ & $\eta_{y}$ & $-2 \xi_{x}$ \\
$a_{1}=2, a_{2}=0$ & $\left(y^{\prime}\right)^{2}$ & $\eta_{y y}$ & $-2 \xi_{x y}$ \\
$a_{1}=1, a_{2}=0$ & $y^{\prime}$ & $2 \eta_{x y}$ & $-\xi_{x x}$ \\
$a_{1}=0, a_{2}=0$ & 1 & $\eta_{x x}$ & \\
$a_{1}=1, a_{2}=1$ & $y^{\prime} y^{\prime \prime}=\left(y^{\prime}\right)^{4}$ & & $-3 \xi_{y}$ \\
$a_{1}=3, a_{2}=0$ & $\left(y^{\prime}\right)^{3}$ & & $-\xi_{y y}$ \\
\hline
\end{tabular}

The coefficients for $\eta^{(1)}$ are easily obtained. Multiplying by $-3\left(y^{\prime}\right)^{2}$ and working modulo the original differential equation, we obtain the following determining equations in Table 2.2.

TABLE 2.2

\begin{tabular}{cccc}
\hline Term & $\eta^{(2)}$ coefficient & $\eta^{(1)}$ coefficient & Determining equation \\
\hline$\left(y^{\prime}\right)^{4}$ & $-3 \xi_{y}$ & $+3 \xi_{y}$ & $0=0$ \\
$\left(y^{\prime}\right)^{3}$ & $\eta_{y}-2 \xi_{x}-\xi_{y y}$ & $-3 \eta_{y}+3 \xi_{x}$ & $-2 \eta_{y}+\xi_{x}-\xi_{y y}=0$ \\
$\left(y^{\prime}\right)^{2}$ & $\eta_{y y}-2 \xi_{x y}$ & $-3 \eta_{x}$ & $\eta_{y y}-2 \xi_{x y}-3 \eta_{x}=0$ \\
$y^{\prime}$ & $2 \eta_{x y}-\xi_{x x}$ & 0 & $2 \eta_{x y}-\xi_{x x}=0$ \\
1 & $\eta_{x x}$ & 0 & $\eta_{x x}=0$ \\
\hline
\end{tabular}

Solving the determining equations, we obtain the general solution for the infinitesimal generator:

$$
\begin{gathered}
\xi(x, y)=c\left(6 x y+y^{3}-6 y^{2}\right)+d\left(2 x+y^{2}\right)+e y+f \\
\eta(x, y)=c(6 y-4 x)+2 d .
\end{gathered}
$$

If we define the coefficients in formula (2.19) as functions of the tuples that satisfied the given condition, we obtain a compact notation.

DEFINITION 2.7. Consider a tuple of $n$ nonnegative integers $\left(c_{1}, c_{2}, \ldots, c_{n}\right)$. Let $r_{i}=$ $\sum_{j=i}^{n} c_{j}$. Define the number

$$
\beta\left(c_{1}, c_{2}, \ldots, c_{n}\right):=\frac{n !}{\left(n-\sum_{i=1}^{n} r_{i}\right) ! \prod_{i=1}^{n}\left(c_{i} ! i^{r_{i}}\right)}
$$

when $\sum_{i=1}^{n} r_{i} \leq n$, and let $\beta\left(c_{1}, c_{2}, \ldots, c_{n}\right):=0$ otherwise. 
Formula (2.19) can then be written in terms of this $\beta$ function as

$$
\begin{aligned}
\eta^{(n)}= & \sum_{\left(b_{1}, b_{2}, \ldots, b_{n}\right)} \beta\left(b_{1}, \ldots, b_{n}\right) \cdot\left(y^{\prime}\right)^{b_{1}} \cdots\left(y^{(n)}\right)^{b_{n}} \cdot \eta_{x^{n-\sum r_{i}} y^{r_{1}}} \\
& -\sum_{\left(b_{1}, b_{2}, \ldots, b_{n}\right)} \frac{\sum r_{i}}{n+1} \beta\left(b_{1}, \ldots, b_{n}, 0\right) \cdot\left(y^{\prime}\right)^{b_{1}} \cdots\left(y^{(n)}\right)^{b_{n}} \cdot \xi_{x^{n+1-\sum r_{i}} y^{r_{1}-1}} .
\end{aligned}
$$

Combining both terms in one summation, we obtain the following.

COROLLARY 2.8. The prolongations formula in (2.19) can be written as

$$
\eta^{(n)}=\sum_{\left(b_{1}, b_{2}, \ldots, b_{n}\right)} \gamma\left(b_{1}, b_{2}, \ldots, b_{n}\right) \cdot\left(y^{\prime}\right)^{b_{1}} \cdots\left(y^{(n)}\right)^{b_{n}}
$$

where

$$
\begin{aligned}
& \gamma\left(b_{1}, b_{2}, \ldots, b_{n}\right) \\
& \quad=\beta\left(b_{1}, \ldots, b_{n}\right) \eta_{x^{n-\sum r_{i}} y^{r_{1}}}-\frac{\sum r_{i}}{n+1} \beta\left(b_{1}, \ldots, b_{n}, 0\right) \xi_{x^{n+1-\sum r_{i}} y^{r_{1}-1}} .
\end{aligned}
$$

Note 2.9. This formula provides a direct calculation of the coefficients of the derivative monomials in the prolongation of the vector field. This, together with the equation itself, is used now to set up the determining equations. We do not need to display the entire prolongation, but we can write directly the coefficients by computing the $\beta$ and $\gamma$ functions.

\section{Other examples}

EXAMPLE 3.1. Calculate the coefficient of $\left(y^{\prime}\right)^{2}\left(y^{(5)}\right)$ in the eighth-prolongation of a vector field. Using our formulas, the coefficient $\alpha_{1}$ is given by

$$
\begin{aligned}
\alpha_{1} & =\gamma(2,0,0,0,1,0,0,0) \\
& =\beta(2,0,0,0,1,0,0,0) \eta_{x y y y}-\frac{7}{9} \beta(2,0,0,0,1,0,0,0,0) \xi_{x x y y} \\
& =\frac{8 !}{(1) !(2) ! 1^{3} 2^{1} 3^{1} 4^{1} 5^{1}} \eta_{x y y y}-\frac{7}{9} \frac{9 !}{(2) !(2) ! 1^{3} 2^{1} 3^{1} 4^{1} 5^{1}} \xi_{x x y y} \\
& =168 \eta_{x y y y}-588 \xi_{x x y y} .
\end{aligned}
$$

Similarly, the coefficient of $\left(y^{\prime \prime}\right)^{4}$ will be

$$
\begin{aligned}
\alpha_{2} & =\gamma(0,4,0,0,0,0,0,0) \\
& =\beta(0,4,0,0,0,0,0,0) \eta_{y y y y}-\frac{4}{9} \beta(0,4,0,0,0,0,0,0,0) \xi_{x y y y} \\
& =\frac{8 !}{(0) !(4) ! 1^{4} 2^{4}} \eta_{y y y y}-\frac{4}{9} \frac{9 !}{(1) !(4) ! 1^{4} 2^{4}} \xi_{x y y y} \\
& =105 \eta_{y y y y}-420 \xi_{x x y y}
\end{aligned}
$$


and the coefficient of $\left(y^{\prime \prime}\right) y^{(7)}$ is

$$
\begin{aligned}
\alpha_{3} & =\gamma(0,1,0,0,0,0,1,0) \\
& =0-\frac{9}{9} \beta(0,1,0,0,0,0,1,0,0) \xi_{y} \\
& =-\frac{9 !}{(0) !(1) ! 1^{2} 2^{2} 3^{1} 4^{1} 5^{1} 6^{1} 7^{1}} \xi_{y} \\
& =-36 \xi_{y} .
\end{aligned}
$$

EXAMPLE 3.2. As another example, consider the equation $(\Delta=) 4 y^{2} y^{(3)}-18 y y^{\prime} y^{\prime \prime}$ $+15\left(y^{\prime}\right)^{3}=0$. The third prolongation applied to the equation gives

$$
4 y^{2} \eta^{(3)}-18 y y^{\prime} \eta^{(2)}+\left(45\left(y^{\prime}\right)^{2}-18 y y^{\prime \prime}\right) \eta^{\prime}+\left(8 y y^{(3)}-18 y^{\prime} y^{\prime \prime}\right) \eta=0 .
$$

We compute directly the coefficients of the third prolongations of $X=(\xi(x, y), \eta(x$, $y)$ ) in terms of the monomials $\left(y^{\prime}\right)^{b_{1}}\left(y^{\prime \prime}\right)^{b_{2}}\left(y^{3}\right)^{b_{3}}$ modulo $\Delta=0$; see Table 3.1.

TABLE 3.1

\begin{tabular}{ll}
\hline Term & Determining equation \\
\hline 1 & $4 y^{2} \eta_{x x x}=0$ \\
$y^{\prime \prime}$ & $4 y^{2} \eta_{x x x}-18 y \eta_{x}=0$ \\
$y^{\prime}$ & $12 y^{2} \eta_{x x y}-4 y^{2} \xi_{x x x}-18 y \eta_{x x}=0$ \\
$\left(y^{\prime}\right)^{2}$ & $12 y^{2} \eta_{x y y}-12 y^{2} \xi_{x x y}-36 y \eta_{x y}+18 y \xi_{x x}+45 \eta_{x}=0$ \\
$\left(y^{\prime}\right)^{3}$ & $4 y^{2} \eta_{y y y}-12 y^{2} \xi_{x y y}+30 \eta_{y}-(30 / y) \eta-18 y \eta_{y y}+36 y \xi_{x y}=0$ \\
$\left(y^{\prime}\right)\left(y^{\prime \prime}\right)$ & $4 y^{2} \eta_{y y}-36 y^{2} \xi_{x y}-18 y \eta_{y}+18 \eta=0$ \\
$\left(y^{\prime}\right)^{4}$ & $-4 y^{2} \xi_{y y y}+15 \xi_{y}+18 y \xi_{y y}=0$ \\
$\left(y^{\prime}\right)^{2}\left(y^{\prime \prime}\right)$ & $-24 y^{2} \xi_{y y}=0$ \\
\hline
\end{tabular}

Looking for nontrivial solutions, the last two equations and the third equation in Table 3.1 imply $\eta_{x}=0$, reducing the system to

$$
\begin{gathered}
\xi_{x x}=0 \\
2 y^{2} \eta_{y y y}+15 \eta_{y}-\left(\frac{15}{y}\right) \eta-9 y \eta_{y y}=0 \\
2 y^{2} \eta_{y y}-9 y \eta_{y}+9 \eta=0 .
\end{gathered}
$$

Since $\eta_{x}=0$, we solve the equations and obtain the infinitesimal generators

$$
\begin{aligned}
& \xi(x, y)=a x+b, \\
& \eta(x, y)=c y^{29 / 6}+d y+e .
\end{aligned}
$$


EXAMPLE 3.3. Consider the equation $y^{(3)}=(3 / 2)\left(\left(y^{\prime \prime}\right)^{2} / y^{\prime}\right)$. The third prolongation applied to the equation gives

$$
y^{\prime} \eta^{(3)}-3 y^{\prime \prime} \eta^{(2)}+\left(y^{(3)}\right) \eta^{\prime}=0
$$

We compute the coefficients of the prolongation of $X=(\xi(x, y), \eta(x, y))$ in terms of the monomials $\left(y^{\prime}\right)^{b_{1}}\left(y^{\prime \prime}\right)^{b_{2}}\left(y^{3}\right)^{b_{3}}$ modulo the equation, multiply it by $y^{\prime}$, and obtain Table 3.2.

TABLE 3.2

\begin{tabular}{ll}
\hline Term & Determining equation \\
\hline$\left(y^{\prime}\right)^{2}$ & $\eta_{x x x}=0$ \\
$\left(y^{\prime}\right)^{2} y^{\prime \prime}$ & $-3 \eta_{x y}=0$ \\
$\left(y^{\prime}\right)^{3}$ & $3 \eta_{x x y}-\xi_{x x x}=0$ \\
$\left(y^{\prime}\right)^{4}$ & $3 \eta_{x y y}-3 \xi_{x x y}=0$ \\
$\left(y^{\prime}\right)^{5}$ & $\eta_{y y y}-3 \xi_{x y y}=0$ \\
$\left(y^{\prime}\right)^{3}\left(y^{\prime \prime}\right)$ & $3 \xi_{x y}=0$ \\
$y^{\prime} y^{\prime \prime}$ & $-3 \eta_{x x}=0$ \\
$\left(y^{\prime}\right)^{2}\left(y^{\prime \prime}\right)^{2}$ & $-(3 / 2) \xi_{y}=0$ \\
$\left(y^{\prime}\right)^{6}$ & $-\xi_{y y y}=0$ \\
$\left(y^{\prime}\right)^{4}\left(y^{\prime \prime}\right)$ & $-3 \xi_{y y}=0$ \\
$\left(y^{\prime \prime}\right)^{2}$ & $(3 / 2) \eta_{x}=0$ \\
\hline
\end{tabular}

Since $\xi_{y}=0, \eta_{x}=0$, the system reduces to $\xi_{x x x}=0, \eta_{y y y}=0$. Thus, the point symmetries of the equation are given by $\xi(x, y)=a x^{2}+b x+c, \eta(x, y)=d y^{2}+e y+f$.

EXAMPLE 3.4. Consider the equation $y^{(6)}=y^{2}$. To find the symmetries, we need to find the sixth prolongation, which can take several pages to write. It is obvious that $X=1 \partial x$ is a symmetry, but is it the only one? Our formulas will help determine this without finding the sixth prolongation of the vector field.

Applying the infinitesimal operator, we obtain

$$
\eta^{(6)}-2 y \eta=0
$$

We look selectively at the determining equations. First, find the one that does not depend on derivatives, namely,

$$
\alpha_{1} \eta_{x x x x x x}-2 y \eta=0
$$

Our formulas give directly the value of $\alpha_{1}=1$.

Since the original equation depends only on the sixth derivative, we look for terms depending on the derivative of $\xi$. The lowest derivative of $\xi$ occurs for $\xi_{x^{7-q} y^{r_{1}-1}}$ when 
$q=7$ and $r_{1}=2$. Thus consider the term

$$
\alpha_{2} \xi_{y} y^{(4)} y^{(2)}
$$

of $J$-order 7. Our formulas give $\alpha_{2}=1$ and produce the determining equation $\xi_{y}=0$.

As a third equation, we consider terms involving $\eta$ and $\xi$ with lowest-order derivatives, for $q=6, r_{1}=1$. This term corresponds to

$$
\alpha_{3} \eta_{y}-\alpha_{4} \xi_{x}=0
$$

The coefficients are calculated by Corollary 2.5, $\alpha_{3}=1, \alpha_{4}=-6$, and we have produce three determining equations

$$
\eta_{x x x x x x}-2 y \eta=0, \quad \xi_{y}=0, \quad \eta_{y}+6 \xi_{x}=0 .
$$

The last two equations produce $\eta=-6 y f^{\prime}(x)+g(x)$. Replacing this into the first equation and using linear independence, we obtain $g(x)=0, f(x)=c$. Thus $\eta=0$ and $\xi=c$ which indicate that $X=\partial x$ is the only symmetry of the differential equation.

Note 3.5. An interesting combinatorial question refers to how many nonzero terms will be in the prolongation, for which we need to know how many $n$-tuples $\left(b_{1}, b_{2}, \ldots, b_{n}\right)$ of nonnegative integers satisfy the condition $\sum_{i=1}^{n} r_{i}=\sum_{i=1}^{n} i b_{i} \leq n$. This is a number theory question, which we leave for the reader to find an answer for.

ACKNOWLEDGMENT. We would like to thank the referees for their comments and corrections of this article.

\section{REFERENCES}

[1] J. Draisma, Recognizing the symmetry type of O.D.E.s, J. Pure Appl. Algebra 164 (2001), no. 1-2, 109-128.

[2] G. Emanuel, Solution of Ordinary Differential Equations by Continuous Groups, Chapman \& Hall/CRC, Florida, 2000.

[3] H. Stephani, Differential Equations, Their Solution Using Symmetries, Cambridge University Press, Cambridge, 1989.

Ricardo Alfaro: Department of Mathematics, University of Michigan-Flint, Flint, MI 48502, USA E-mail address: ralfaro@umflint.edu

Jim Schaeferle: Department of Mathematics, University of Michigan-Flint, Flint, MI 48502, USA E-mail address: Schaefer1e@comcast. net 


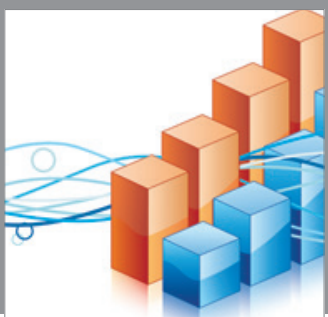

Advances in

Operations Research

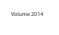

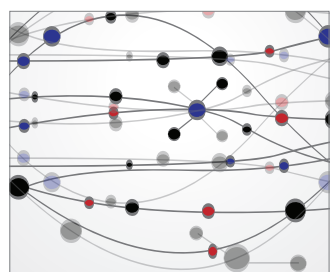

\section{The Scientific} World Journal
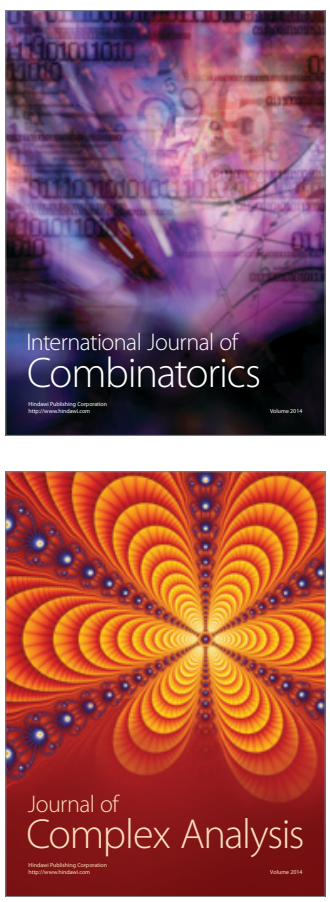

International Journal of

Mathematics and

Mathematical

Sciences
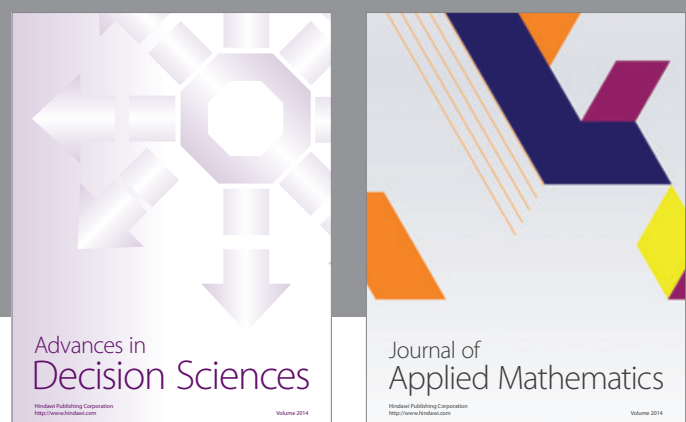

Journal of

Applied Mathematics
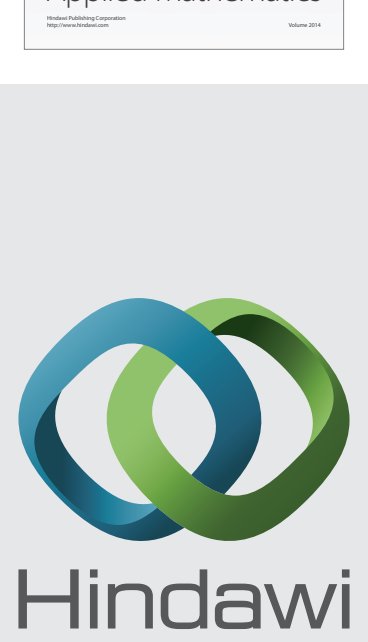

Submit your manuscripts at http://www.hindawi.com
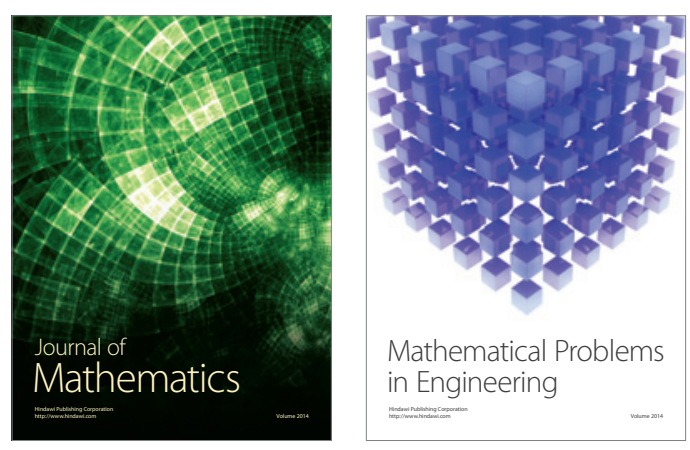

Mathematical Problems in Engineering
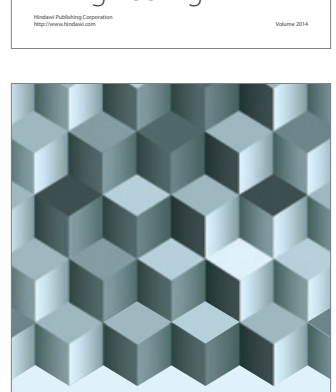

Journal of

Function Spaces
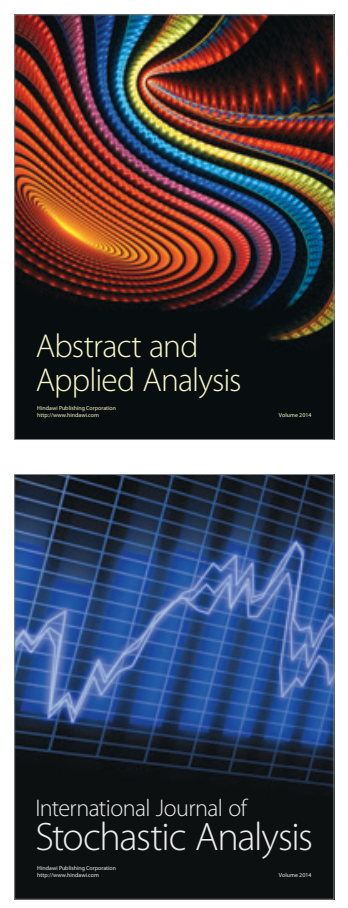

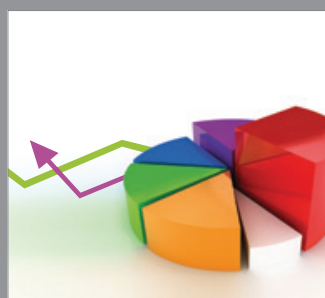

ournal of

Probability and Statistics

Promensencen
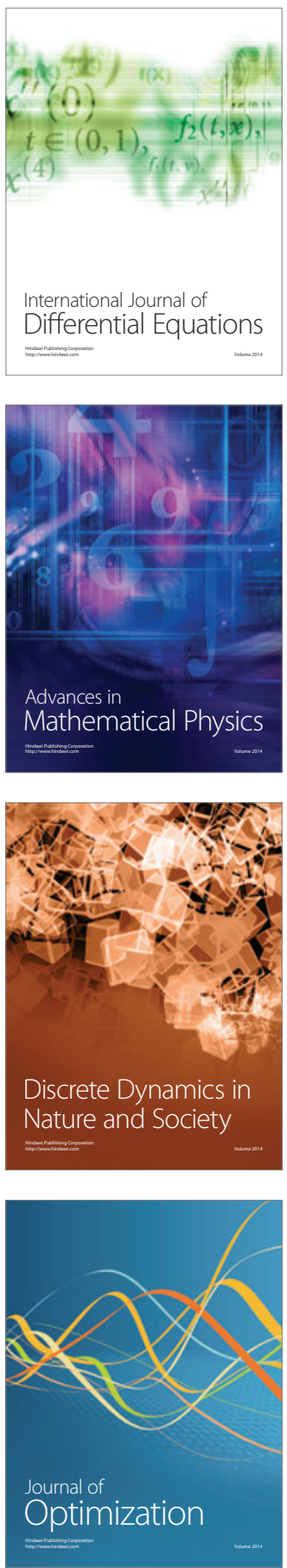